\title{
Actitud hacia la ética profesional en estudiantes de postgrado de una facultad de medicina
}

\section{Attitude to professional ethics in medicine postgraduate students of a medical school}

\author{
Manuel Izaguirre ${ }^{1, a}$, Martha Vera ${ }^{2, b}$, Luis Podestáa ${ }^{1, c}$, Deysi Córdova ${ }^{3, d}$ \\ ${ }^{1}$ Departamento Académico de Pediatría, Facultad de Medicina, Universidad Nacional Mayor de San Marcos. Lima, Perú. \\ ${ }^{2}$ Departamento Académico de Enfermería, Facultad de Medicina, Universidad Nacional Mayor de San Marcos. Lima, Perú. \\ ${ }^{3}$ Facultad de Medicina, Universidad Nacional Mayor de San Marcos. Lima, Perú. \\ ${ }^{a}$ Doctor en educación, ORCID https://orcid.org/ 0000-0002-0768-7402 \\ ${ }^{\mathrm{b}}$ Doctora en ciencias de la salud, ORCID: https://orcid.org/0000-0001-8811-8959 \\ 'Doctor en ciencias de la salud, ORCID https://orcid.org/0000-0003-0122-8835 \\ ${ }^{\mathrm{d}}$ Estudiante de la maestría en salud ocupacional y ambiental
}

\section{An Fac med. 2020;81(1):52-7 / DOI: https://doi.org/10.15381/anales.v81i1.16972}

\section{Correspondencia:}

Manuel Izaguirre Sotomayor

manuel.izaguirre@gmail.com

Recibido: 5 de noviembre 2019 Aceptado: 8 de febrero 2020

Publicación en línea: 31 de marzo 2020

Conflictos de interés: Los autores declaran no tener conflictos de interés

Fuente de financiamiento: Vicerectorado de Investigación y Posgrado, Universidad Nacional Mayor de San Marcos, 2018.

Citar como: Izaguirre M, Vera M, Podestá L, Córdova D. Actitud hacia la ética profesional en estudiantes de postgrado de medicina. An Fac med. 2020;81(1):52-7. DOI: https://doi. org/10.15381/anales.v8111.16972

\section{Resumen}

Introducción. La profesión médica ha dejado de ser respetada por la sociedad como consecuencia del comportamiento no profesional de muchos de sus miembros, por la deshumanización, la corrupción y por la cosificación de la medicina al priorizar lo económico y el bienestar personal a lo social. Objetivos. Conocer la actitud hacia la ética profesional en estudiantes del postgrado de la Facultad de Medicina de la Universidad Nacional Mayor de San Marcos. Métodos. Se realizó un estudio descriptivo, transversal, de enfoque cuantitativo, utilizando como instrumento la escala de actitudes sobre ética profesional. Resultados. La actitud hacia la ética profesional fue positiva en el $81,5 \%$ e indiferente en el 18,5\%. La actitud positiva hacia la ética profesional en las competencias cognitiva y técnica, sociales, éticas y afectivo emocionales fue de $88,8 \%, 69,2 \%, 80 \%$ y $87,7 \%$, respectivamente. Conclusión. La actitud hacia la ética profesional fue positiva en el $81,5 \%$ e indiferente en el $18,5 \%$.

Palabras clave: Actitud; Ética Profesional; Estudiantes del Área de la Salud; Programas de Posgrado en Salud (fuente: DeCS BIREME).

\section{Abstract}

Introduction. The medical profession has ceased to be respected by society as a consequence of the unprofessional behavior of many of its members, due to dehumanization, corruption and the reification of medicine by prioritizing the economic and personal well-being over the social. Objective. To know the attitude towards professional ethics in postgraduate students of the Faculty of Medicine of the Universidad Nacional Mayor de San Marcos. Methods. A descriptive, cross sectional study with a quantitative approach was carried out, using the scale of attitudes on professional ethics as an instrument. Results. The attitude towards professional ethics was positive in $81,5 \%$ and indifferent in $18,5 \%$. The positive attitude towards professional ethics in cognitive and technical, social, ethical, and emotional-emotional competencies was $88,8 \%, 69,2 \%$, $80 \%$, and $87,7 \%$, respectively. Conclusion. The attitude towards professional ethics was positive in $81,5 \%$ and indifferent in $18,5 \%$.

Keywords: Attitude; Ethics, Professional; Students, Health Occupations; Health Postgraduate Programs (source: MeSH NLM). 


\section{INTRODUCCIÓN}

La ética profesional se define como el comportamiento ético guiado por los valores de autonomía, responsabilidad y competencia profesional ${ }^{(1)}$. La profesión médica ha dejado de ser respetada porque el conocimiento médico y la tecnología, aunado al comportamiento no profesional de algunos han erosionado el respeto ganado por la medicina ${ }^{(2)}$, al haber priorizado lo económico y el bienestar personal a lo social, contraviniendo estándares éticos del profesionalismo y tradición médica ${ }^{(3)}$.

Por tanto, la ética en la profesión es imprescindible al darle la capacidad de identificar lo correcto de lo incorrecto, dilema que todo profesional afronta al tomar decisiones, aspirando a hacer lo adecuado en beneficio de sus pacientes. La ética es lo que le da sentido a la medicina para no hacer daño ni ser maleficente en el ejercicio profesional. Nunca priorizar el beneficio económico al bienestar del paciente por ser una persona con derecho y protección moral y legal, que obliga reconocerle autoridad moral para participar en las decisiones terapéuticas ${ }^{(4)}$, evitando la "desprofesionalización" al perderse el compromiso con el bienestar y el interés del paciente, toda vez que el aprendizaje ético sólo será posible con el desarrollo de los valores institucionales, siendo insuficiente que las universidades introduzcan la ética en el currículo en un intento por humanizar la profesión médica ${ }^{(5)}$.

El ser profesional de ciencias de la salud obliga a no brindar una medicina de vanguardia para una minoría privilegiada, como tampoco solo curar y no prevenir, sino, convertirse en un profesional con voluntad y coraje que cumple con los estándares éticos, convirtiéndose en protector de su autonomía y de sus valores morales y personales ${ }^{(6)}$.

Estudios previos realizados para conocer la actitud hacia la ética profesional en estudiantes de postgrado muestran diferentes porcentajes de actitud positiva hacia la ética profesional, tanto en postgrado relacionado al área de la salud ${ }^{(7-10)}$ como en otros campos profesionales ${ }^{(11,12)}$.

La presente investigación tuvo como objetivo determinar la actitud hacia la éti- ca profesional de estudiantes de postgrado de ciencias de la salud, con la finalidad de contribuir a la enseñanza ética y las decisiones frente a los conflictos éticos.

\section{MÉTODOS}

Se realizó un estudio observacional, descriptivo, transversal, de enfoque cuantitativo. La muestra fue por conveniencia, constituida por 260 estudiantes del postgrado (maestrías y doctorados) de la Facultad de Medicina de la Universidad Nacional Mayor de San Marcos, el año 2018. Se utilizó la Escala de Likert modificada sobre la ética profesional para 4 competencias: cognitivas y técnicas, sociales, éticas, y afectivo-emocionales, validada por Hirsch ${ }^{(13)}$. Las 55 preguntas comprendieron 5 categorías de respuesta con valores 1 a 5. Para la calificación, se asignó un puntaje inverso. Se definió actitud negativa para los puntajes 1 ó 2 ; indiferentes para el puntaje 3 y positiva para los puntajes 4 ó 5 . Se calcularon promedios para cada competencia, dimensión y constructo, así como para el total.

Se garantizó la confidencialidad de los datos y se solicitó el consentimiento informado a cada uno de los participantes.

\section{RESULTADOS}

De los 260 participantes del estudio, el grupo etario predominante fue 30 a 39 años. La actitud hacia la ética profesional fue positiva en el $81,5 \%$ e indiferente en el 18,5\%. Dentro de las competencias de la ética profesional, el 88,8\% tuvieron una actitud positiva hacia las competencias cognitivas y técnicas, en las dimensiones: conocimiento, formación, preparación y competencia profesional, alcanzando la máxima valoración el constructo "me produce satisfacción la adquisición de nuevos conocimientos profesionales" (Tabla 1).

El 69,2\% de los participantes tuvieron una actitud positiva hacia las competencias sociales, alcanzando la máxima valoración la dimensión ser trabajador, en el constructo "trabajar con ahínco es parte de mi realización personal (Tabla 2).
En las competencias éticas, el $80 \%$ tuvieron una actitud positiva, alcanzando la máxima valoración la dimensión responsabilidad, en el constructo "guardar la confidencialidad es importante en el ejercicio profesional". La menor valoración estuvo en la dimensión actuar con la idea de prestar un mejor servicio a la sociedad, en el constructo "Lo que me interesa preferentemente es ganar dinero y prestigio" (Tabla 3).

El $87.7 \%$ tuvieron una actitud positiva hacia las competencias afectivo emocionales, alcanzando la máxima valoración las dimensiones identificarse con la profesión, en el constructo "Es un gran logro hacer profesionalmente lo que más me gusta" y en la capacidad emocional, para el constructo "Hacer lo correcto profesionalmente me permite estar en paz conmigo mismo".

En resumen, la actitud positiva hacia la ética profesional le correspondió mayoritariamente a los que integraban la competencia cognitivas y técnicas y la menor a los que integraban las competencias sociales. El 20\% de los estudiantes tuvieron una actitud indiferente hacia las competencias éticas (Tabla 4).

\section{DISCUSIÓN}

La investigación pretende aproximarse a la conducta ética y deliberación moral de los profesionales de la salud para realizar o no una actividad que se convierte en obligación de la experiencia ética, que implica la realización de valores ${ }^{(14)}$. Que la mayoría tuviera una actitud positiva hacia las competencias cognitivas y técnicas, resultados concordantes con los obtenidos en otros estudios ${ }^{(7,8,9,12)}$, indicarían que está relacionada con la responsabilidad de adquirir nuevos conocimientos para desarrollar sus actividades dentro de un marco de cientificidad, creatividad, actitud reflexiva, acorde a los avances de la ciencia y la tecnología, para construir permanentemente el saber desde principios éticos en búsqueda de la verdad ${ }^{(15)}$. Toda vez que responde a la responsabilidad ética el prevenir la negligencia o delito, al brindar una atención de calidad basada en la evidencia científica, respetando los principios éticos y actuando en concordancia con su 
Tabla 1. Actitud de los estudiantes de posgrado según competencias cognitivas y técnicas de la ética profesional. Facultad de Medicina, UNMSM, 2018.

\begin{tabular}{|c|c|c|c|c|c|c|}
\hline \multirow{2}{*}{ Competencias } & \multirow{2}{*}{ Dimensiones } & \multirow{2}{*}{ Constructo } & \multicolumn{3}{|c|}{ Actitud } & \multirow{2}{*}{ Total } \\
\hline & & & Negativa & Indiferente & Positiva & \\
\hline \multirow{13}{*}{$\begin{array}{l}\text { I. COGNITIVAS Y } \\
\text { TÉCNICAS }\end{array}$} & \multirow{2}{*}{$\begin{array}{l}\text { a) Conocimiento, } \\
\text { formación, } \\
\text { preparación y } \\
\text { competencia } \\
\text { profesional }\end{array}$} & $\begin{array}{l}\text { Me gusta tratar con profesionales que tengan buenos } \\
\text { conocimientos científicos }\end{array}$ & $0,4 \%$ & $3,1 \%$ & $96,5 \%$ & $100 \%$ \\
\hline & & $\begin{array}{l}\text { Me produce satisfacción la adquisición de nuevos conocimientos } \\
\text { profesionales }\end{array}$ & $0,4 \%$ & $0,4 \%$ & $99,2 \%$ & $100 \%$ \\
\hline & \multirow{4}{*}{$\begin{array}{l}\text { b) Formación } \\
\text { continua }\end{array}$} & $\begin{array}{l}\text { La puesta al día en los conocimientos es imprescindible para ser un } \\
\text { buen profesional }\end{array}$ & $0 \%$ & $3,1 \%$ & $96,9 \%$ & $100 \%$ \\
\hline & & $\begin{array}{l}\text { Si no estoy preparándome continuamente no puedo resolver } \\
\text { nuevas situaciones y problemas profesionales }\end{array}$ & $5,8 \%$ & $23,8 \%$ & $70,4 \%$ & $100 \%$ \\
\hline & & $\begin{array}{l}\text { Me producen desconfianza aquellos que creen saberlo todo en la } \\
\text { profesión }\end{array}$ & $14,6 \%$ & $32,3 \%$ & $53,1 \%$ & $100 \%$ \\
\hline & & No me agrada tener que prepararme continuamente & $8,1 \%$ & $5,8 \%$ & $86,2 \%$ & $100 \%$ \\
\hline & \multirow{6}{*}{$\begin{array}{l}\text { c) Innovación y } \\
\text { superación }\end{array}$} & $\begin{array}{l}\text { Estoy dispuesto (a) a ocupar tiempo en actualizar mis } \\
\text { conocimientos sobre algún aspecto de mi profesión }\end{array}$ & $0 \%$ & $3,5 \%$ & $96,5 \%$ & $100 \%$ \\
\hline & & Estoy dispuesto (a) a dedicar dinero a mi formación & $1,5 \%$ & $4,2 \%$ & $94,2 \%$ & $100 \%$ \\
\hline & & Me preocupa que pueda ejercer mi profesión de un modo rutinario & $8,1 \%$ & $18,5 \%$ & $73,5 \%$ & $100 \%$ \\
\hline & & Creo necesario hacer cosas nuevas para avanzar en la profesión & $1,2 \%$ & $5,8 \%$ & $93,1 \%$ & $100 \%$ \\
\hline & & Disfruto cuando tengo que aprender algo nuevo & $0,4 \%$ & $1,5 \%$ & $98,1 \%$ & $100 \%$ \\
\hline & & $\begin{array}{l}\text { Considero conveniente aceptar el riesgo de equivocarme con tal de } \\
\text { mejorar mi actividad profesional }\end{array}$ & $6,5 \%$ & $21,2 \%$ & $72,3 \%$ & $100 \%$ \\
\hline & $\begin{array}{l}\text { d) Competencias } \\
\text { técnicas }\end{array}$ & $\begin{array}{l}\text { Para mi buen ejercicio profesional no puedo limitarme a desarrollar } \\
\text { sólo las habilidades técnicas }\end{array}$ & $3,5 \%$ & $7,7 \%$ & $88,8 \%$ & $100 \%$ \\
\hline
\end{tabular}

responsabilidad moral que obliga a no ser solo un buen profesional, sino un profesional bueno que rinde cuenta de sus actos a su consciencia ${ }^{(16)}$.

Que la actitud positiva hacia la dimensión competencias sociales tuviera la más baja valoración, coincidente con los resul- tados obtenidos por otros autores (7,8,10,12), se correlacionaría con el trabajo en equipo, brindar atención integral y de calidad desde la interdisciplinariedad donde cada profesional, desarrolla actividades especializadas con ahínco a través de la interrelación y compañerismo que constituye parte de su realización personal. En este sentido, evidenciaría su compromiso con su responsabilidad legal al cumplir los códigos de ética para evitar ser negligente o cometer delito, al estar obligado a ser justo y no maleficente. Sin embargo, casi un tercio tuvo una actitud indiferente hacia las competencias

Tabla 2. Actitud de los estudiantes de posgrado según competencias sociales de la ética profesional. Facultad de Medicina, UNMSM, 2018.

\begin{tabular}{|c|c|c|c|c|c|c|}
\hline Competencias & Dimensiones & Constructo & Negativa & $\begin{array}{l}\text { Actitud } \\
\text { Indiferente }\end{array}$ & Positiva & Total \\
\hline \multirow{9}{*}{ II. SOCIALES } & \multirow{2}{*}{$\begin{array}{l}\text { a) Compañerismo } \\
\text { y relaciones }\end{array}$} & $\begin{array}{l}\text { Me siento mejor cuando tengo presente las necesidades } \\
\text { profesionales de mis compañeros }\end{array}$ & $3,8 \%$ & $17,7 \%$ & $78,5 \%$ & $100 \%$ \\
\hline & & $\begin{array}{l}\text { Me gusta que mis compañeros de trabajo valoren } \\
\text { positivamente mi buen trato con las personas }\end{array}$ & $1,2 \%$ & $11,2 \%$ & $87,7 \%$ & $100 \%$ \\
\hline & \multirow{3}{*}{ b) Comunicación } & $\begin{array}{l}\text { No necesito ponerme en lugar de mis clientes para } \\
\text { comprender sus necesidades }\end{array}$ & $24,6 \%$ & $15,8 \%$ & $59,6 \%$ & $100 \%$ \\
\hline & & $\begin{array}{l}\text { La solución de los problemas sociales es un asunto técnico } \\
\text { que hace innecesario escuchar a los ciudadanos }\end{array}$ & $18,5 \%$ & $15,8 \%$ & $65,8 \%$ & $100 \%$ \\
\hline & & $\begin{array}{l}\text { Considero que puedo resolver importantes cuestiones } \\
\text { profesionales escuchando a los demás }\end{array}$ & $3,5 \%$ & $16,5 \%$ & $80 \%$ & $100 \%$ \\
\hline & \multirow{2}{*}{$\begin{array}{l}\text { c) Saber trabajar } \\
\text { en equipo }\end{array}$} & $\begin{array}{l}\text { Prefiero trabajar en equipo porque el resultado es de más } \\
\text { alta calidad }\end{array}$ & $2,3 \%$ & $12,3 \%$ & $85,4 \%$ & $100 \%$ \\
\hline & & $\begin{array}{l}\text { Se equivocan las instituciones que no promueven el trabajo } \\
\text { en equipo de sus profesionales }\end{array}$ & $7,3 \%$ & $11,9 \%$ & $80,8 \%$ & $100 \%$ \\
\hline & \multirow[t]{2}{*}{ d) Ser trabajador } & $\begin{array}{l}\text { Estoy convencido (a) de que para ser un buen profesional } \\
\text { tendré que hacer algún tipo de sacrificio }\end{array}$ & $3,5 \%$ & $15.8 \%$ & $80,8 \%$ & $100 \%$ \\
\hline & & Trabajar con ahínco es parte de mi realización personal & $1,9 \%$ & $3.5 \%$ & $94,6 \%$ & $100 \%$ \\
\hline
\end{tabular}


Tabla 3. Actitud de los estudiantes de posgrado según competencias éticas. Facultad de Medicina, UNMSM, 2018.

\begin{tabular}{|c|c|c|c|c|c|c|}
\hline \multirow{2}{*}{ Competencias } & \multirow{2}{*}{ Dimensiones } & \multirow{2}{*}{ Constructo } & \multicolumn{3}{|c|}{ Actitud } & \multirow{2}{*}{ Total } \\
\hline & & & Negativa & Indiferente & Positiva & \\
\hline \multirow{23}{*}{ III. ÉTICAS } & \multirow{3}{*}{ a) Responsabilidad } & $\begin{array}{l}\text { Estoy dispuesto (a) a asumir las consecuencias de mis errores } \\
\text { profesionales }\end{array}$ & $1,5 \%$ & $3,8 \%$ & $94,6 \%$ & $100 \%$ \\
\hline & & $\begin{array}{l}\text { Guardar la confidencialidad es importante en el ejercicio } \\
\text { profesional }\end{array}$ & $0 \%$ & $1,2 \%$ & $98,8 \%$ & $100 \%$ \\
\hline & & $\begin{array}{l}\text { El cumplimiento a tiempo de mis compromisos profesionales } \\
\text { es importante }\end{array}$ & $0,8 \%$ & $2,7 \%$ & $96,5 \%$ & $100 \%$ \\
\hline & \multirow{2}{*}{ b) Honestidad } & $\begin{array}{l}\text { Para no cometer errores en mi ejercicio profesional debo ser } \\
\text { consciente de los límites de mis conocimientos y habilidades }\end{array}$ & $2,7 \%$ & $8,5 \%$ & $88,8 \%$ & $100 \%$ \\
\hline & & $\begin{array}{l}\text { Debo ganarme la confianza de mis clientes y usuarios actuando } \\
\text { con honestidad }\end{array}$ & $1,9 \%$ & $6,2 \%$ & $91,9 \%$ & $100 \%$ \\
\hline & \multirow{4}{*}{$\begin{array}{l}\text { c) Ética profesional } \\
\text { y personal }\end{array}$} & $\begin{array}{l}\text { Hay decisiones éticas tan importantes en el ejercicio de } \\
\text { mi profesión que no pueden dejarse sólo a criterio de las } \\
\text { organizaciones }\end{array}$ & $1,5 \%$ & $12,7 \%$ & $85,8 \%$ & $100 \%$ \\
\hline & & $\begin{array}{l}\text { Mientras la ciencia y la tecnología sigan avanzando no es } \\
\text { necesario preocuparnos de sus consecuencias }\end{array}$ & $17,7 \%$ & $11,9 \%$ & $70,4 \%$ & $100 \%$ \\
\hline & & $\begin{array}{l}\text { La formación en ética me puede ser necesaria para enfrentar } \\
\text { conflictos en el trabajo profesional }\end{array}$ & $1,5 \%$ & $7,3 \%$ & $91,2 \%$ & $100 \%$ \\
\hline & & $\begin{array}{l}\text { Considero imprescindible tomar muy en cuenta los aspectos } \\
\text { éticos en el ejercicio de mi profesión }\end{array}$ & $1,2 \%$ & $4,2 \%$ & $94,6 \%$ & $100 \%$ \\
\hline & \multirow{6}{*}{$\begin{array}{l}\text { d) Actuar con la } \\
\text { idea de prestar el } \\
\text { mejor servicio a la } \\
\text { sociedad }\end{array}$} & $\begin{array}{l}\text { A los profesionales no les corresponde la solución de los } \\
\text { problemas sociales }\end{array}$ & $18,5 \%$ & $10,4 \%$ & $71,2 \%$ & $100 \%$ \\
\hline & & $\begin{array}{l}\text { Para ser un buen profesional no puedo ignorar los problemas } \\
\text { de la sociedad en la que vivo }\end{array}$ & $3,8 \%$ & $8,1 \%$ & $88,1 \%$ & $100 \%$ \\
\hline & & $\begin{array}{l}\text { En la profesión, ayudar a los demás es más importante que } \\
\text { alcanzar el éxito }\end{array}$ & $5,4 \%$ & $20 \%$ & $74,6 \%$ & $100 \%$ \\
\hline & & Lo que me interesa preferentemente es ganar dinero y prestigio & $17,7 \%$ & $19,2 \%$ & $63,1 \%$ & $100 \%$ \\
\hline & & $\begin{array}{l}\text { De nada sirve un trabajo bien hecho si no contribuye a ayudar } \\
\text { a los demás }\end{array}$ & $3,5 \%$ & $10,4 \%$ & $86,2 \%$ & $100 \%$ \\
\hline & & Seleccioné mi carrera para ser útil a las personas & $2,3 \%$ & $10 \%$ & $87,7 \%$ & $100 \%$ \\
\hline & \multirow{2}{*}{ e) Respeto } & $\begin{array}{l}\text { Me gusta tratar con profesionales que toman en cuenta mi } \\
\text { opinión }\end{array}$ & $1,2 \%$ & $6,2 \%$ & $92,7 \%$ & $100 \%$ \\
\hline & & $\begin{array}{l}\text { Respeto las opiniones de los clientes y usuarios sobre mi } \\
\text { trabajo profesional }\end{array}$ & $0 \%$ & $7,3 \%$ & $92,7 \%$ & $100 \%$ \\
\hline & \multirow{6}{*}{$\begin{array}{l}\text { f) Actuar con } \\
\text { sujeción a } \\
\text { principios } \\
\text { morales y valores } \\
\text { profesionales }\end{array}$} & No estoy dispuesto (a) a ejercer mi profesión sólo por dinero & $12,3 \%$ & $12,3 \%$ & $75,4 \%$ & $100 \%$ \\
\hline & & $\begin{array}{l}\text { No es preciso que conozca y practique los valores de mi } \\
\text { profesión para poder ejercerla }\end{array}$ & $19,6 \%$ & $9,2 \%$ & $71,2 \%$ & $100 \%$ \\
\hline & & $\begin{array}{l}\text { El éxito profesional no significa nada, si no me permite ser una } \\
\text { mejor persona }\end{array}$ & $5 \%$ & $7,7 \%$ & $87,3 \%$ & $100 \%$ \\
\hline & & $\begin{array}{l}\text { Un buen profesional debe darse tiempo para evaluar las } \\
\text { consecuencias de sus acciones }\end{array}$ & $0,8 \%$ & $4,2 \%$ & $95,0 \%$ & $100 \%$ \\
\hline & & $\begin{array}{l}\text { La coherencia con los principios éticos es más importante que } \\
\text { ganar dinero }\end{array}$ & $4,6 \%$ & $10 \%$ & $85,4 \%$ & $100 \%$ \\
\hline & & Transmito mis propios valores a través del ejercicio profesional & $0 \%$ & $4,2 \%$ & $95,8 \%$ & $100 \%$ \\
\hline
\end{tabular}

sociales, que evidenciaría la irresponsabilidad legal, la crisis moral de la sociedad y la indiferencia hacia los principios éticos que la sociedad ha construido ${ }^{(14)}$.

La gramática moral permitiría articular respuestas lentas evaluadas por el componente racional de las conductas morales, directas, y rápidas estimadas por su componente emocional, convirtiéndose en sustrato básico de lo moral, además, de ser predecibles y estructuradas en cualquier cultura, al margen de sus valores y creencias específicas ${ }^{(15,17)}$. Decidir sobre lo bueno y lo malo, percibido por los sentimientos y convertido en valores personales, "generaría respuestas homeostáticas entre lo eficiente e ineficiente", obligando a desarrollar normas convertidas en códigos o leyes para el adecuado ejercicio profesional. Es un hecho, que los circuitos relacionados con las emociones se activan en la toma de decisiones éticas ${ }^{(17)}$.

Que las competencias éticas estuvieran en el tercer lugar en la valoración de la actitud positiva, como lo estuvieron en las investigaciones de Hirsch y Navia ${ }^{(7,8)}$, y 
Tabla 4. Porcentaje de estudiantes por tipo de actitud hacia la ética profesional, según competencias. Facultad de Medicina, UNMSM, 2018.

\begin{tabular}{|c|c|c|c|c|c|c|}
\hline \multirow[b]{2}{*}{ Competencias } & \multicolumn{3}{|c|}{ Actitud } & \multirow[b]{2}{*}{ Total } & \multirow[b]{2}{*}{ Número de ítems } & \multirow[b]{2}{*}{ Informantes } \\
\hline & $\begin{array}{l}\text { Totalmente } \\
\text { negativo }\end{array}$ & Indiferente & $\begin{array}{l}\text { Totalmente } \\
\text { positivo }\end{array}$ & & & \\
\hline Total & $0 \%$ & $18,5 \%$ & $81,50 \%$ & $100 \%$ & 55 & 260 \\
\hline I. Competencias cognitivas y técnicas & $0 \%$ & $11,2 \%$ & $88,8 \%$ & $100 \%$ & 13 & 260 \\
\hline II. Competencias sociales & $0 \%$ & $30,8 \%$ & $69,2 \%$ & $100 \%$ & 9 & 260 \\
\hline III. Competencias éticas & $0 \%$ & $20 \%$ & $80 \%$ & $100 \%$ & 23 & 260 \\
\hline $\begin{array}{l}\text { IV. Competencias afectivo - } \\
\text { emocionales }\end{array}$ & $0 \%$ & $12,3 \%$ & $87,7 \%$ & $100 \%$ & 10 & 260 \\
\hline
\end{tabular}

Cada ítem tuvo un valor en un rango de 1 a 5 . Totalmente negativo incluye a informantes que respondieron 1 u 2 en todas las preguntas; Totalmente positivo incluye a informantes que respondieron 4 o 5 en todas las preguntas; Indiferente comprende a los otros casos.

contrariamente a lo encontrado por PérezCastro y Páramo ${ }^{(10,11)}$ en donde ocupaba el primer lugar, se sustentaría en la ética práctica que analiza "los hechos, posteriormente los valores que están soportados por esos hechos, y finalmente los deberes que se desprenden de la realización de los valores" que son cualidades y, si es intrínseco, vale por su naturaleza y por sí mismo, soportados en los hechos. En este sentido, los hechos, valores y deberes se construyen social e históricamente ${ }^{(15,17)}$.

Las normas y leyes generadas por respuestas emocionales básicas y por el binomio bueno-malo han posibilitado la convivencia grupal, al establecer normas de conducta. Estas respuestas morales son producidas por la activación de áreas corticales profundas del lóbulo temporal, especialmente de la amígdala y la ínsula ${ }^{(17)}$.

Valorar es un acto individual y colectivo que se construye históricamente; que "el dinero es paradigma de valores instrumentales... que el significado de la vida solo se adquiere por valores intrínsecos, que muchas veces son desplazados por los valores instrumentales... siendo la función de la ética educar en la gestión razonable o prudente de la vida" (18).

Los entrevistados fueron conscientes de la importancia de la confidencialidad, de cumplir compromisos, asumir las consecuencias de los errores, respetar las opiniones de los pacientes al ser hechos que generan valores y deberes que tienen que cumplir, por ser principio ético que los obliga. Sin embargo, a más de los dos tercios le interesaba ganar dinero y prestigio como correlato de la deshumanización de la medicina que "ha erosio- nado la posición históricamente respetada de la medicina" y al hecho de conciliar el currículo explícito y el oculto los habría llevado a "desarrollar una profesionalidad no reflexiva" ${ }^{(2,3)}$, como consecuencia de considerar a la medicina bien económico y enfocarse en la tecnología y acto médico y no en la persona ${ }^{(19)}$.

El funcionamiento moral respondería a "un sistema de módulos paralelos... que elaboran la información de modo competitivo, dan respuestas priorizadas y jerárquicas, condicionadas al estímulo y a la experiencia previa" que pueden ser utilitaristas o morales, influenciadas por la cultura existente ${ }^{(15,20)}$. En este sentido, más de los cuatro quintos de los entrevistados manifestaron que hacer lo que les gusta es el mayor logro profesional, hacer lo correcto le permite estar tranquilo con su conciencia moral que se daría cuenta de lo que es bueno y lo que es malo y gracias a la empatía, sentir "estados funcionales neuronales de los semejantes" (20,4), lo que explicaría la valoración baja al hecho de tener aspiraciones, pero no una ambición desmedida.

La competencia afectivo emocional ocupó el segundo lugar de valoración positiva, como lo estuvo en otros estudios ${ }^{(7-10)}$, alcanzando la máxima valoración hacia las dimensiones identificarse con la profesión, en el constructo "es un gran logro hacer profesionalmente lo que más me gusta" y en la capacidad emocional, para el constructo "hacer lo correcto profesionalmente me permite estar en paz conmigo mismo".

Finalmente, uno de cada cinco tuvo una actitud indiferente hacia las competencias éticas, superando a lo encontrado por Hirsch ${ }^{(7)}$. Obliga a las universidades asumir que la ética profesional debería ser pilar fundamental de su accionar en el ámbito universitario, al hacerse imperativo que las decisiones y el comportamiento se sustenten en la moral que es universal y en la ética que rigen la conducta encaminada al bien común y sus valores ${ }^{(15,20)}$, toda vez que lo técnicamente bien realizado, no siempre es éticamente bueno ${ }^{(14)}$

Todo profesional necesita tener competencia profesional, técnica y ética para que todo acto, decisión o comportamiento sean guiadas por valores en bien de la sociedad ${ }^{(5)}$, dado que la no solución de los conflictos éticos condiciona deterioro de la calidad de atención, al no ser consciente de su responsabilidad ética y jurídica ${ }^{(14)}$. Toda decisión tiene que ser responsable y moral, tomada libremente en su condición de ser, un ser moral que tiene que rendir cuenta de sus actos ante sí y la sociedad, dado que sus decisiones se convierten en la razón moral de su conciencia, más ante la crisis moral existente es indispensable que las universidades incorporen la ética como eje transversal en el currículo como respuesta a la demanda de la sociedad para que formen profesionales con valores éticos ${ }^{(6,14)}$.

La muestra no probabilística y las respuestas subjetivas fueron las limitaciones del presente estudio, lo que plantea valorar las actitudes, interpretando decisiones tomadas ante situaciones hipotéticas planteadas.

En conclusión, en la muestra estudiada, la actitud hacia la ética profesional fue positiva en el $81,5 \%$ e indiferente en el $18,5 \%$. 


\section{REFERENCIAS BIBLIOGRÁFICAS}

1. Hirsch A. Elementos significativos de la ética profesional. Reencuentro. 2003;(38):8-15.

2. Stobo J, Blank L. Project Professionalism: Staying Ahead of the Wave. American Board of Internal Medicine; 2001.

3. Bloom SW. Professionalism in the practice of medicine. Mt Sinai J Med. 2002;69(6):398-403.

4. Polo MA. Ética Profesional. Revista de Investigación de la Facultad de Ciencias Administrativas UNMSM. 2003;6(12):69-78

5. Bolivar A. El lugar de la ética profesional en la formación universitaria. Revista Mexicana de Investigación Educativa. 2005;10(24):93-123.

6. Pellegrino ED, Thomasma DC. The virtues in medical practice. Oxford University Press: New York; 1993.

7. Hirsch A, Pérez J. Actitudes y ética profesiona en estudiantes de posgrado en la Universidad de Valencia y la UNAM. Reencuentro. 2005;(43).
8. Navia C, Hirsch A. Ética profesional en estudiantes de posgrado en dos universidades mexicanas. Revista Electrónica de Investigación Educativa. 2015;17(1):100-115.

9. Chávez G. Rasgos y actitudes de Ética Profesional en estudiantes de posgrado de la UAT y de la UANL. X Congreso Nacional de Investigación Educativa área 6: educación y valores.; 2019.

10. Pérez-Castro J. La Ética Profesional en la formación universitaria en México. EDETANIA. 2015;47:93-107.

11. Páramo M, Torrecilla M, Garcia C, Straniero C. Estudio de las actitudes hacia la ética profesional en estudiantes de grado y posgrado de psicología. Investigaciones en Psicologia. 2018;(23):65-74.

12. Izarra D. Actitudes de docentes y estudiantes de posgrado en educación. aproximación a su identidad y ética profesional. EDETANIA. 2019;55:117-129.

13. Hirsch A. Construcción de una escala de actitudes sobre ética profesional. Revista Electrónica de Investigación Educativa. 2005;7(1):1-14.
14. Gracia D. La deliberación moral: el método de la ética clínica. Med Clin (Barc). 2001;117: 1823.

15. Thomas Star D. Measuring Medical Professionalism. Oxford University Press Academic. Printed in the United States of America; 2006.

16. Wagner P, Perales A, Armas R, Codas O, De los Santos R, Elio Calvo D, et al. Bases y perspectivas latinoamericanas sobre Medicina y Salud Centradas en la Persona. An Fac med. 2015;76(1):63-70. DOI: 10.15381/anales.v76i1.11077

17. Álvaro-González L. Neurética (I): circuitos morales en el cerebro normal. Rev Neurol. 2014; 58 (5):225-233.

18. Álvarez-Díaz J. Neuroética del trasplante de cuerpo. Rev Neurol. 2018;67(12):505-512.

19. Álvarez-Díaz JA. Hechos, valores, deberes...y neuronas. Rev Neurol. 2013;(57): 576

20. Gracia D. Construyendo valores. Ed. Triacastela Madrid; 2013. 